\title{
Robotic Radical Prostatectomy and Nerve Grafting: Does it Actually Work?
}

\author{
Goonewardene SS ${ }^{1 *}$, Persad R ${ }^{2}$, Gillatt $\mathrm{D}^{3}$ \\ ${ }^{1}$ University of Warwick, Guys and St Thomas Hospitals London \\ ${ }^{2}$ North Bristol NHS Trust, Bristol \\ ${ }^{3}$ Mcquarrie University, Sydney
}

Received: February 01, 2016; Accepted: February 22, 2016; Published: March 30, 2016

*Corresponding author: Goonewardene SS, University of Warwick, Guys and St Thomas Hospitals London, Email: ssg7727@yahoo.co.uk

Robotic radical prostatectomy is now the gold standard for patients with localised prostate cancer. In the setting of unfavorable prostate pathology, some men may require resection of one or both cavernous nerves at the time of prostatectomy. Potency rates in this subset of men are expectedly dismal [1]. With increasing numbers of younger men being diagnosed with prostate cancer and subsequently undergoing radical prostatectomy, there is an increasing focus on quality of life postoperatively, especially potency [2]. Nerve-grafting surgery after resection of neuro-vascular bundles during radical prostatectomy is one of the promising resolutions for dilemma between cancer control and functional preservation [3]. There have been a number of articles recently on nerve grafting for robotic radical prostatectomy. We aim to review the evidence for whether this actually works and gives a good degree of erectile function.

2 and 5 year follow ups of patient reported Health Related Quality of Life (HRQOL) after open Radical Prostatectomy (RP) [4] demonstrated persistent sexual dysfunction and urinary incontinence post-operatively [4]. As a substitute for injured or excised cavernosal nerves which may occur during radical prostatectomy, nerve grafting procedures have been tried to provide a substitute [5].

Cavernous nerve graft is an option for sexually active men requiring cavernous nerve resection for cancer control during pelvic surgery [6]. Several reports demonstrated favourable outcomes of autologous nerve grafting with respect to sexual function [7]. Cavernous nerve reconstruction may improve the rate of sexual function recovery after pelvic surgery [6]. However, sexual bother has not decreased within this cohort. This was further confirmed, as implantation of the allogenic nerve graft in patients undergoing non nerve-sparing radical prostatectomy has been shown to be safe [7]. Implantation of this allogenic nerve graft appears to make sense in the reconstructive peripheral surgery [8]. However, functional results were awaited.

Nonrandomized studies of Unilateral Nerve-Sparing (UNS) Radical Prostatectomy (RP) have reported improved recovery of erectile function, if the sacrificed cavernous nerve is reconstructed with a Sural Nerve Graft (SNG) [9]. However, the addition of SNG to a UNS RP did not improve potency at 2 years following surgery [9].

Interposition nerve grafting offers a scaffold for orderly nerve regeneration and may elaborate neurotrophic agents that foster the restorative process [10]. Traditionally, autologous sural nerve has been the graft of choice and has been employed primarily during open radical prostatectomy [10]. However, sural nerve harvest is often associated with donor site morbidity and may be time inefficient [10].

With nerve-sparing techniques, patients undergoing a radical prostatectomy may avoid the morbidity of erectile dysfunction [2]. Certain patients who are not candidates for nerve-sparing procedures may be eligible for nerve interposition grafts [2]. While bilateral cavernosal nerve grafting after radical prostatectomy has shown efficacy, the effect of unilateral nerve grafting following prostatectomy remains unclear. Unilateral sural nerve grafting is a feasible and well-tolerated approach for patients who must undergo wide resection of a NVB [2]. While men do show a decrease in their IIEF score, $76 \%$ are able to achieve penetration following surgery [2].

Use of robotics for microsurgery may provide advantages in terms of offering magnification, motion scaling and additional surgical arms in a stable ergonomic platform [11]. Previously, the microsurgeon could perform only two standard microsurgical procedures a day due to fatigue limitations using the standard microscope [11]. With the aid of dual robotic systems, the same microsurgeon has been able to routinely perform up to ten microsurgical procedures a day due to the ergonomic advantages of the robot [11]. This was confirmed by [8] however this paper concluded robotic assisted microsurgery is still evolving [12]. A number of microsurgical procedures were successfully performed [12]. However, the overall primary benefits appear to be improved surgical efficiency and less reliance on a skilled surgical assistant [12]. 
Regenerative medicine for the penis was introduced to restore structural and functional abnormalities [5]. To achieve functional restoration of the corpus cavernosum, cell-based therapy was proposed using bioengineered replacement of cavernosal tissue [5]. Even though there are controversies surrounding ethical aspects of the issue, stem cell therapy with adult stem cells or progenitor cells could be a promising approach for the management of erectile dysfunction [5]. On a molecular level again, restoration of erectile function is paralleled by an increase of neuronal Nitric oxide expression in rats [13]. Further experiments will determine the physiological role of neuronal nitric oxide in erectile nerve repair processes [13].

In conclusion, the true benefit of interposition nerve grafting has yet to be determined [10]. The robotic operating platform offers filtration of tremor and outstanding optics that lends itself to microsurgical reconstruction such as interposition grafting [1]. Likewise, new acellular allografts are more robust than autologous nerves and confer surgical efficiency [10]. However, there is still controversy over its use because of the lack of any large, blinded trials, the anatomy of the cavernous nerves and the necessity of excising the neurovascular bundles (especially bilaterally) [2]. In addition, the results achieved with nerve grafting, a procedure not without significant morbidity and mortality, do not exceed those produced by surgeons carrying out nerve-sparing procedures [2].

With kind regards,

SS Goonewardene, R Persad and D Gillatt declare they have no conflicts of interest and nothing to disclose.

\section{References}

1. White WM, Kim ED. Interposition Nerve Grafting During Radical Prostatectomy: Cumulative Review and Critical Appraisal of Literature. Urology. 2009;74(2):245-250. doi: 10.1016/j.urology.2008.12.059.

2. Hanson GR, Borden LS, Backous DD, Bayles SW, Corman JM. Erectile function following unilateral cavernosal nerve replacement. Can J Urol. 2008;15(2):3990-3993.

3. Sugimoto M, Tsunemori H, Kakehi Y. Health-related quality of life evaluation in patients undergoing cavernous nerve reconstruction during radical prostatectomy. Jpn J Clin Oncol. 2009;39(10):671-676. doi: 10.1093/jjco/hyp082.

4. Namiki S, Arai Y. Health-related quality of life in men with localized prostate cancer: Review Article. International Journal of Urology. 2010;17(2):125-138.

5. Park KS. Regenerative medicine in erectile dysfunction. Journal of Men's Health. 2011;8:S103.

6. Matsuoka Y, Tohi Y, Suenaga T, Go S, Hayashida Y, Kato T, et al. Health-related quality of life in patients who underwent autologous cavernous nerve reconstruction during radical prostatectomy; longterm observation. Journal of Sexual Medicine Conference: 14th Biennial Meeting of the Asia Pacific Society for Sexual Medicine Kanazawa Japan Conference Start. 10(pp 242):242.

7. Kwol M, Vollmer C, Horstmann M, Padevit C, John H. Nerve grafting with an allograft during radical prostatectomy: Prospective, randomised, blinded study. Urology. 2013;52(1):S115.

8. Vollmer C, Horstmann M, Padevit C, John H. 344 Nerve grafting with an allograft during radical prostatectomy: Prospective, randomized, blinded study. European Urology. Supplements. 2012;11(1):e344.

9. Davis JW, Chang DW, Chevray P, Wang R, Shen Y, Wen S, et al. Randomized Phase II Trial Evaluation of Erectile Function after Attempted Unilateral Cavernous Nerve-Sparing Retropubic Radical Prostatectomy With Versus Without Unilateral Sural Nerve Grafting for Clinically Localized Prostate Cancer. European Urology. 2009;55(5):1135-1144.

10. White W, Stewart A, Hatcher P, Klein F, Kim E. Robotic interposition nerve grafting using an acellular allograft nerve. American Urological Association. 2011;(1):e492.

11. Parekattil SJ, Priola KB, Atalah H, Cohen MS. Robotic assisted microsurgery: The initial 293 case experience. Journal of Endourology. 2010;24:A274.

12. Gudeloglu A, Brahmbhatt J, Lee D, Priola K, Parekattil S. Evolution of robotic microsurgery: Single center, prospective database, 692 case experiences. Journal of Endourology. 2012;26:A406-A407.

13. Schlenker B, Matiasek K, Saur D, Gratzke C, Bauer RM, Herouy Y, et al. Effects of cavernous nerve reconstruction on expression of nitric oxide synthase isoforms in rats. BJU International. 2010;106(11):17261731. 